\title{
Dynamics of somatic cell count patterns as a proxy for transmission of mastitis pathogens
}

\author{
Gunnar Dalen, ${ }^{1,2 *}$ Amira Rachah, ${ }^{1}$ Håvard Nørstebø, ${ }^{1,2}$ Ynte H. Schukken, ${ }^{3,4,5}$ and Olav Reksen ${ }^{1}$ \\ ${ }^{1}$ Norwegian University of Life Sciences, Faculty of Veterinary Medicine, Department of Production Animal Clinical Sciences, PO Box 369 sentrum, \\ $\mathrm{N}-0102$ Oslo, Norway \\ ${ }^{2}$ TINE SA, PO Box 58, N-1430 Ås, Norway \\ ${ }^{3}$ GD Animal Health, Arnsbergstraat 7, 7400 AA Deventer, the Netherlands \\ ${ }^{4}$ Department of Animal Sciences, Wageningen University, 6708 PB Wageningen, the Netherlands \\ ${ }^{5}$ Department of Population Medicine and Diagnostic Sciences, Cornell University, Ithaca, NY 14850
}

\section{ABSTRACT}

Management of udder health is particularly focused on preventing new infections. Data from the DeLaval Online Cell Counter (DeLaval, Tumba, Sweden) may be used in forecasting to improve decision support for improved udder health management. It provides online cell counts (OCC) as a proxy for somatic cell counts from every milking at the cow level. However, these values are typically too insensitive and nonspecific to indicate subclinical intramammary infection (IMI). Our aim was to describe and evaluate use of dynamic transmission models to forecast subclinical IMI episodes using milk cultures or changes in OCC patterns over time. The latter was expressed by an elevated mastitis risk variable. Data were obtained from the dairy herd of the Norwegian University of Life Sciences (Oslo, Norway). In total, 173 cows were sampled monthly for bacteriological milk culture during a 17-mo study period and 5,330 quarter milk samples were cultured. Mastitis pathogens identified were assigned to 1 of 2 groups, Pat 1 or Pat 2. Pathogens from which a high cell count would be expected during a subclinical IMI episode were assigned to the Pat 1 group. Pathogens not in the Pat 1 group were assigned to the Pat 2 group. Staphylococcus epidermidis, Staphylococcus aureus, and Streptococcus dysgalactiae were the most common Pat 1 pathogens. Corynebacterium bovis, Staphylococcus chromogenes, and Staphylococcus haemolyticus were the most common Pat 2 pathogens. The OCC were successfully recorded from 82,182 of 96,542 milkings. The current study included 324 subclinical IMI episodes. None of the mastitis pathogens demonstrated a basic reproduction number $\left(\mathrm{R}_{0}\right)>1$. Patterns of OCC change

Received April 23, 2019.

Accepted August 18, 2019.

*Corresponding author: gunnar.dalen@nmbu.no related to an episode of Pat 1 subclinical IMI at specificity levels of 80,90 , and $95 \%$ at sensitivity levels of 69,59 , and $48 \%$ respectively, demonstrated an $\mathrm{R}_{0}>1$. An existing infection was significant for transmission for several Pat 2 pathogens, but only for Staphylococcus aureus and Staphylococcus epidermidis among Pat 1 pathogens. Dynamic transmission models showed that patterns of OCC change related to an episode of Pat 1 subclinical IMI were significantly related to the same pattern occurring in susceptible cows at specificity levels of 80,90 , and $99 \%$ at sensitivity levels of 69,48 , and $8 \%$, respectively. We conclude that changes in herd prevalence of subclinical IMI can be predicted using dynamic transmission models based on patterns of OCC change. Choice of specificity level depends on management goals and tolerance for false-positive alerts.

Key words: intramammary infection, transmission, somatic cell count, online cell count

\section{INTRODUCTION}

Management of udder health is particularly focused on preventing new infections (Ruegg, 2017). Common management approaches apply standard operating procedures using historical information (Østerås and Sølverød, 2009; Scherpenzeel et al., 2016), which yields slow-moving improvement. Therefore, real-time detection and management of transmission of subclinical IMI may improve management of udder health.

An IMI is defined as being present when a quarter is infected with a bacterial species (Berry and Meaney, 2006). In many but not all cases, an IMI may be identified based on an increase in SCC (Sargeant et al., 2001). This inflammatory response, often caused by an IMI, is defined as mastitis (Djabri et al., 2002). When clinical symptoms occurs, this is defined as clinical mastitis; when there is an increase in SCC but no clinical signs occur, this is defined as subclinical mastitis (IDF, 2011). 
To some extent, SCC values can be used for surveillance of IMI (Schukken et al., 2003). For reasons of surveillance and precision diagnostics, the industry has advanced toward developing sensors specifically for udder health. One of these sensors is the DeLaval Online Cell Counter (DeLaval International AB, Tumba, Sweden). Using this sensor, we can obtain repeated online cell counts (OCC) at the cow level. These data may be implemented in automated detection systems for management of udder health in automatic milking systems (AMS). Based on smoothed OCC data, Sørensen et al. (2016) created an elevated mastitis risk (EMR) indicator to detect cases of clinical mastitis. This EMR indicator is a continuous variable (from 0 to 1 ), where values close to 0 indicate a low risk of mastitis and higher values, approaching 1, indicate an increased risk of clinical mastitis (Sørensen et al., 2016).

Dalen et al. (2019) demonstrated that the EMR indicator can be used to detect subclinical mastitis episodes in individual cows. However, when every milking is a potential detection event, the diagnostic test properties are insufficiently sensitive for direct application in a decision-support tool (Dalen et al., 2019). Therefore, the interpretation and use of OCC during lactation should be improved in decision-support tools for dairy farmers.

Compartmental transmission models are powerful tools for understanding infection dynamics by providing predictions about the potential transmission of infections and the efficacy of control measures (Magal and Ruan, 2008; Otto and Day, 2011). Pathogen-specific transmission patterns have been described for major and minor mastitis pathogens (Zadoks et al., 2002; White et al., 2006; Reksen et al., 2012). However, these models have not previously been applied to patterns of OCC change associated with subclinical IMI episodes. By modeling the patterns of OCC change associated with subclinical IMI episodes, as a proxy for transmission of pathogens, the underlying infection pressure in the herd can be continuously monitored. When there is an increase in the prevalence of a particular pattern of OCC change, there are probably more cows in the herd that have the potential to transmit mastitis pathogens to susceptible cows. Forecasting future development of this transmission-associated pattern, by dynamic simulation modeling, could be used to determine whether actions should be taken to reduce transmission risk.

The primary aim of this study was to describe and evaluate the possibility of using dynamic transmission models to forecast the herd prevalence of subclinical IMI episodes by exploiting measured changes in OCC patterns over time. Specifically, we first wanted to estimate the transmission parameters of subclinical IMI episodes based on culture results and associated changes in OCC patterns expressed by the EMR. Second, we wanted to evaluate the effect of preexisting episodes on new subclinical IMI episodes defined by culture results or changes in the OCC patterns.

\section{MATERIALS AND METHODS}

\section{Field Study}

This study used data obtained during a 17-mo longitudinal observational study in the research herd at the Norwegian University of Life Sciences (Oslo, Norway). On average, 96 cows were milked 2.6 times a day in 2 identical AMS (Delaval VMS, DeLaval, Tumba, Sweden). Mean OCC and milk production per cow per day were 115,103 cells $/ \mathrm{mL}$ and $27.9 \mathrm{~kg}$, respectively. The farm used standardized mastitis control practices, such as post-milking teat disinfection, selective dry-cow therapy, and monthly milk quality testing in a DHIA program.

The 2 AMS were set to record OCC from every milking during the study period. Quarter milk samples (QMS) were collected monthly from all lactating cows, according to recommended sampling guidelines (Hogan et al., 1999). Samples were frozen and transported to the laboratory, where bacteriological culture was performed according to standard procedures (Hogan et al., 1999). Briefly, $0.01 \mathrm{~mL}$ of milk from each quarter was spread on cattle blood agar plates with esculin and incubated at $37^{\circ} \mathrm{C}$. Plates were read at 24 and $48 \mathrm{~h}$. We used a MALDI-TOF MS Microflex LT system (Bruker Corp., Billerica, MA; Cheuzeville, 2015) for species identification of cultured bacteria. Further details on the study herd, sampling framework, and microbial analyses were previously published (Dalen et al., 2019).

\section{Subclinical IMI Status}

In this study, we investigated subclinical IMI episodes only. Cows treated for clinical mastitis were transferred to a treatment pen without AMS, and we do not have bacteriological samples or OCC records throughout the period of treatment for the clinical mastitis cases. The diagnosis of subclinical IMI was based on bacteriological culture results or using OCC data as a proxy for bacteriological culture results. A cow was considered to have a subclinical IMI episode with an individual mastitis pathogen when meeting at least one of the following criteria: (1) $\geq 1,000 \mathrm{cfu} / \mathrm{mL}$ of a single mastitis pathogen cultured from a single sample in at least 1 quarter, (2) $\geq 500 \mathrm{cfu} / \mathrm{mL}$ of a mastitis pathogen cultured from 2 out of 3 consecutive milk samples from the 
same quarter, or $(3) \geq 100 \mathrm{cfu} / \mathrm{mL}$ of a mastitis pathogen cultured from 3 consecutive milk samples from the same quarter. These definitions are adapted from those of Zadoks et al. (2002).

Because OCC is recorded at the cow level, and our aim was to model transmission of subclinical IMI based on both individual and grouped mastitis pathogens, as well as on patterns of OCC change, we aggregated the bacteriological diagnoses at the quarter level into cowlevel diagnoses. Also, because the same cow could experience a subclinical IMI episode with different mastitis pathogens at the same time, pathogens were divided into 2 groups (Pat $\mathbf{1}$ and Pat 2), according to characteristics of the bacteria. The group of pathogens from which a high cell count would be expected during a subclinical IMI episode was designated Pat 1 , according to Dalen et al. (2019). The pathogens included in the Pat 1 group were Staphylococcus aureus, Streptococcus dysgalactiae, Streptococcus uberis, Enterococcus faecalis, Enterococcus faecium, Lactococcus lactis, Staphylococcus epidermidis, and Staphylococcus simulans (Djabri et al., 2002; Reksen et al., 2008; Simojoki et al., 2009, 2011; Fry et al., 2014). Mastitis pathogens that were not included in Pat 1 were grouped in the Pat 2 category. This included Corynebacterium bovis, Staphylococcus chromogenes, Staphylococcus haemolyticus, Aerococcus viridans, Staphylococcus hominis, Staphylococcus xylosus, and other mastitis pathogens (Dalen et al., 2019). A cow was considered to have a Pat 1 or Pat 2 subclinical IMI when one or more quarters were positive for a Pat 1 or a Pat 2 mastitis pathogen, respectively.

Because sampling was performed monthly, the exact time of infection and cure was not known. Therefore, we used the mid-point estimation method previously described by Zadoks et al. (2002) to calculate the infection period. We defined the start of the subclinical IMI episode as the middle of the time interval between a negative culture and the first positive culture event, and defined the end of the subclinical IMI episode as the middle of the time interval between the last positive culture event and the first negative culture for a quarter defined as cured (Zadoks et al., 2002).

\section{OCC}

Online cell counts were successfully recorded from 82,182 of 96,542 milkings (85\%); the 14,360 missing values were due either to equipment failure or failure to service and refill the OCC unit with reagent. We computed EMR values (as described by Sørensen et al., 2016; Dalen et al., 2019) for all milkings. Statistical analyses were conducted using Stata (Stata SE/14, Stata Corp., College Station, TX). Briefly, the validity of all recorded OCC measurements were checked before logarithmic transformation. We included only milkings from 5 to 305 DIM with a milking interval of 4 to 24 $\mathrm{h}$ and a yield of $\geq 3.5 \mathrm{~kg}$. Also, OCC values of 0 were omitted from further analyses. Lactation-specific OCC curves were calculated for first, second, and third and later lactations using Wood's lactation curve (Wood, 1967). For milkings with missing OCC data, the missing data were replaced with a value given by $95 \%$ of the previous value and $5 \%$ of the lactation-specific OCC curve for the cow at the DIM of the milking with missing data. This way, the OCC curve of cows with missing data approached the lactation-specific OCC curves by $5 \%$ for each milking where OCC was missing (Sørensen et al., 2016).

The ln-transformed OCC data were adjusted for aberrations and drift at the sensor level by single exponential smoothing (Hyndman et al., 2008), before double exponential smoothing of the adjusted OCC values according to Sørensen et al. (2016).

The lactation-specific OCC curves were used for rapid initialization of the double exponential smoothing (Sørensen et al., 2016). The output from the double exponential smoothing (level and trend) were used to calculate EMR values for every milking on a continuous scale from 0 to 1 (Sørensen et al., 2016). Because both the level and trend are used in calculation of the EMR, the underlying historic and current OCC values can be different in 2 cows with the same EMR value. Therefore, we use the term "OCC pattern" to describe the OCC changes associated with EMR values. Furthermore, we used the threshold values from Dalen et al. (2019) to assign cows to a subclinical IMI status, based on the OCC pattern given by the EMR value. Cows were classified as having a subclinical IMI when the EMR value was greater than the threshold. For the 4 patterns of OCC change, the EMR value thresholds were $0.03,0.05,0.08$, and 0.62 at specificity levels of $80,85,90$, and $99 \%$. The corresponding sensitivities for each pattern of OCC change for detection of Pat 1 subclinical IMI were $69,59,48$, and $8 \%$, respectively (Dalen et al., 2019).

\section{Transmission Parameters}

The transmission parameter $(\beta)$ was calculated using Poisson regression (Stata SE/14, Stata Corp.) with number of new episodes of subclinical IMI in each monthly interval $\left(I_{M}\right)$ as the outcome, and offset $\ln \frac{S I}{N}$, where $S=$ cow-days of a susceptible cow, $I=$ cow-days infected, $N=$ total cow-days in each interval (study month), and $\beta^{*}$ is the intercept in the equation 
$\ln \left(I_{M}\right)=\beta^{*}+\ln \frac{S I}{N}$. The transmission coefficient $\beta$ is expressed as $\mathrm{e}^{\beta}$.

We estimated the cure rate $(\alpha)$ using Poisson regression (Stata SE/14, Stata Corp.). As outcome, the number of subclinical IMI episodes cured in each monthly interval $\left(C_{M}\right)$ was used, with $\ln (I)$ as offset. Cure rate $(\alpha)$ is the intercept and $I=$ cow-days infected in each monthly interval (study month) in the equation: $\ln \left(C_{M}\right)$ $=\alpha+\ln I$, where the cure rate $\alpha$ is expressed as $\mathrm{e}^{\alpha}$ and $C_{M}=$ cured subclinical IMI episodes in each monthly interval.

Furthermore, we evaluated population-level transmission dynamics using the basic reproduction number, $\mathbf{R}_{0}$, which is given by the expression $\mathbf{R}_{0}=\frac{\beta}{\mu+\alpha}$. The observed rate of entry and exit of cows to and from the lactation pen is $\mu$, and the duration of infection is the inverse of the cure rate $(\alpha)$.

Variance of $\mathrm{R}_{0}$ was obtained using a log-transformation, where $\ln \left(\mathrm{R}_{0}\right)=\ln \left(\frac{\beta}{\mu+\alpha}\right)$, and then further simplified to $\ln (\beta)-\ln (\mu+\alpha)$. The variance of $\ln \left(\mathrm{R}_{0}\right)$ is then variance $\ln (\beta)+$ variance $\ln (\mu+\alpha)$, assuming no covariance between $\beta$ and $\mu+\alpha$. The individual variances are obtained from the regression equations as described above. An estimate of the variance of $R_{0}$ is then obtained and the standard error (SE) by obtaining the square root of the resulting estimate. Subsequently, we used $\pm 1.96 \times \mathrm{SE}$ to calculate a confidence interval for $\mathrm{R}_{0}$.

Infection dynamics may be studied using the subclinical IMI status of cows, as defined above, or using patterns of OCC change, thereby assuming that patterns of OCC change indicate the presence of a subclinical IMI. Observations from the first $7 \mathrm{~d}$ of each cow were omitted for the calculation of transmission parameters for patterns of OCC change. This was done to allow "burn in" of the EMR status, because the EMR for every cow is, by default, initialized with the lactationspecific OCC curves of the herd (Sørensen et al., 2016), and this is likely to be too low for cows with subclinical IMI. Also, as cows were milked several times each day and the transmission models use cow-days as the time variable, only the first observation of the EMR per day was retained in the transmission model of changes in OCC pattern.

\section{Transmission Models}

We evaluated the transmission dynamics of the different mastitis pathogens separately and for the groups Pat 1 and Pat 2, as well as the 4 different patterns

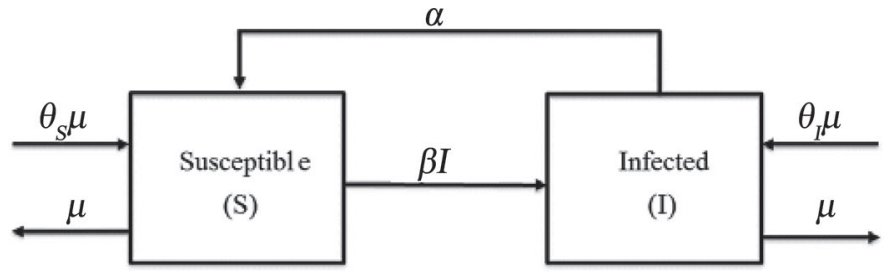

Figure 1. Schematic representation of the mathematical model of transmission of subclinical IMI. The boxes represent the state variables and the arrows represent the flow rates between susceptible (S) and infected (I) states. $\beta=$ transmission parameter, $\beta I$ is the daily rate of new infections, $\alpha=$ daily rate of cured cows; $\mu=$ daily rate of entry and exit of lactating cows. Proportion of cows entering the $\mathrm{S}$ and I compartments are determined by $\theta_{S}$ and $\theta_{I}$, respectively.

of OCC change. We modeled subclinical IMI episodes only. The transmission dynamics of the different pathogens, pathogen groups, and patterns of OCC change were displayed in a Susceptible-Infectious-Susceptible (SIS) model for each pathogen, pathogen group, and change in OCC pattern. The compartmental model describes a population of lactating cows divided into 2 compartments, where S denotes susceptible cows with no subclinical IMI, and I denotes cows with subclinical IMI, where the compartments represent the proportion of lactating cows in each state. Figure 1 illustrates the state transition dynamics.

The following nonlinear ordinary differential equations describe the model mathematically:

$$
\begin{gathered}
\frac{\mathrm{d} S}{\mathrm{~d} t}=-\beta S I+\alpha I+\theta_{S} N \mu-\mu S, \\
\frac{\mathrm{d} I}{\mathrm{~d} t}=\beta S I-\alpha I+\theta_{I} N \mu-\mu I .
\end{gathered}
$$

The parameters $\alpha$ and $\beta$ quantify the transfer rates, where the transmission rate of infection from a cow with subclinical IMI to a susceptible cow is described by $\beta$ (Keeling and Rohani, 2011). The daily rate of cured cows is described by $\alpha$. At any given time, the sum of susceptible and infected cows is represented by $N$. The parameter $\mu$ describes the daily rate of entry and exit of cows to and from the lactation pen. The proportions $\theta_{S}$ and $\theta_{I}$ describe cows entering the lactation pen from the fresh pen to the susceptible or the infectious compartment, respectively.

The difference in $2 \times$ log-likelihood between the model predicting number of new episodes of subclinical IMI with $\ln \frac{S I}{N}$ used as the offset term and the model with only $\ln S$ as the offset was used to evaluate the effect of existing subclinical IMI episodes on the transmission from infected to susceptible cows. The differ- 
ence was evaluated with chi-squared statistics with 1 df.

A nonlinear programming solver of Matlab (MathWorks, Natick, MA), "ode45" solver, was used to solve the nonlinear ordinary equations of the SIS model. This approach is based on the Runge-Kutta method (Dormand and Prince, 1980). The parameter values used in the numerical simulations of the SIS model were obtained from the statistical analysis.

\section{RESULTS}

\section{Field Study}

We collected 5,330 QMS from a total of 257 lactations in 173 cows. Each cow was sampled, on average, 8 times (range 1 to 16). The cows entered the study at an average of 38 DIM (range 4 to 269 DIM). Bacteria were cultured from 1,222 samples, with 1 and 2 pathogens cultured in 1,152 and 67 samples, respectively. According to our criteria for evaluation of contamination, 3 samples were excluded from the analysis. We recorded 16 veterinary treatments for clinical mastitis during the study period. Mastitis pathogens were found in 222 lactations in 155 cows. The most common pathogens found were Staph. epidermidis, C. bovis, Staph. chromogenes, Staph. aureus, and Staph. haemolyticus.
A detailed overview of the distribution of microbial diagnoses can be found in Dalen et al. (2019). According to our definition of subclinical IMI, there were 324 subclinical IMI episodes during the study period.

\section{Estimation of Transmission Parameters}

From the statistical analyses, we obtained transmission parameter $\beta$, cure rate $\alpha$, and daily rate of cows leaving and entering the lactation pen $\mu$; based on these parameters, we calculated $\mathrm{R}_{0}$ for each pathogen and pathogen group, and for the pathogen-proxy pattern of OCC change. The distribution of subclinical IMI episodes and the associated transmission parameters for the different pathogens, pathogen groups, and patterns of OCC change are shown in Table 1. None of the individual mastitis pathogens nor the grouped Pat 1 subclinical IMI or Pat 2 subclinical IMI were found to have an $\mathrm{R}_{0}>1$. However, patterns of OCC change with a specificity of 80,85 , and $90 \%$ for Pat1 subclinical IMI had an $\mathrm{R}_{0}>1$. The average duration of the subclinical IMI episodes, as given by the inverse of the cure rate $\alpha$, is shown for each pathogen and group in Table 2. The duration of subclinical IMI episodes was significantly lower for the 4 patterns of OCC change than for the Pat 1 subclinical IMI episodes.

Table 1. Transmission parameters ${ }^{1}$ for subclinical IMI with individual and grouped (Pat 1 and Pat 2 ) mastitis pathogens and for 4 online cell count (OCC) patterns with different levels of specificity for detection of Pat 1 subclinical IMI episodes

Subclinical IMI with pathogen,

group, or pattern $\quad \mathrm{N}^{2}$

Staphylococcus epidermidis

Corynebacterium bovis

Staphylococcus chromogenes

Staphylococcus aureus

Staphylococcus haemolyticus

Aerococcus viridans

Enterococcus faecalis, Enterococcus faecium,

and Lactococcus lactis

Streptococcus dysgalactiae

Staphylococcus simulans

Staphylococcus hominis

Streptococcus uberis

Staphylococcus xylosus

Other

Pat 1

Pat 2

Elevated mastitis risk (EMR) $80 \%$ specificity $^{3}$

EMR $85 \%$ specificity $^{4}$

EMR 90\% specificity $^{5}$

EMR $99 \%$ specificity $^{6}$
$\mathrm{N}^{2} \quad \beta(95 \% \mathrm{CI})$

$0.0088(0.0063-0.0123)$

$0.0179(0.0139-0.0231)$

$0.0024(0.0012-0.0046)$

$0.0093(0.0060-0.0142)$

$0.0061(0.0036-0.0106)$

$0.0254(0.0158-0.0408)$

$0.0023(0.0009-0.0061)$

0.0065 (0.0036-0.0117)

$0.0294(0.0132-0.0655)$

$0.0030(0.0007-0.0119)$

$0.0043(0.0036-0.0064)$

$0.0165(0.0099-0.0273)$

$0.0069(0.0053-0.0091)$

$0.0048(0.0036-0.0064)$

$0.1368(0.1310-0.1428)$

$0.1465(0.1377-0.1559)$

$0.1768(0.1662-0.1881)$

$0.2692(0.2380-0.3045)$ $\alpha(95 \% \mathrm{CI})$

$0.0080(0.0058-0.0111)$

$0.0074(0.0052-0.0107)$

$0.0038(0.0023-0.0062)$

$0.0078(0.0050-0.0123)$

$0.0062(0.0037-0.0105)$

$0.0203(0.0121-0.0343)$

$0.0022(0.0008-0.0058)$

$0.0077(0.0045-0.0130)$

$0.0046(0.0017-0.0123)$

$0.0242(0.0101-0.0582)$

$0.0029(0.0007-0.0116)$

$0.0086(0.0021-0.0342)$

$0.0139(0.0080-0.0239)$

$0.0048(0.0036-0.0064)$

$0.0070(0.0056-0.0087)$

$0.0889(0.0851-0.0928)$

$0.1039(0.0976-0.1107)$

$0.1368(0.1285-0.1456)$

$0.2575(0.2275-0.2914)$
$\mathrm{R}_{0}(95 \% \mathrm{CI})$

$0.71(0.45-1.14)$

$1.53(0.98-2.39)$

$0.30(0.13-0.67)$

$0.76(0.41-1.42)$

$0.58(0.27-1.24)$

$1.03(0.51-2.09)$

$0.35(0.09-1.41)$

$0.54(0.25-1.19)$

$1.03(0.31-3.38)$

$0.41(0.06-2.93)$

$0.34(0.03-3.71)$

$0.91(0.43-1.90)$

$0.76(0.51-1.13)$

$0.43(0.32-0.58)$

$1.45(1.36-1.54)$

$1.34(1.22-1.46)$

$1.24(1.14-1.36)$

$1.02(0.86-1.22)$

${ }^{1} \beta=$ transmission parameter; $\alpha=$ daily rate of cured cows; $\mathrm{R}_{0}=$ basic reproduction number.

${ }^{2}$ Number of subclinical IMI episodes.

${ }^{3}$ Pattern of OCC change with $80 \%$ specificity and $69 \%$ sensitivity for detection of Pat 1 subclinical IMI episodes.

${ }^{4}$ Pattern of OCC change with $85 \%$ specificity and $59 \%$ sensitivity for detection of Pat 1 subclinical IMI episodes.

${ }^{5}$ Pattern of OCC change with $90 \%$ specificity and $48 \%$ sensitivity for detection of Pat 1 subclinical IMI episodes.

${ }^{6}$ Pattern of OCC change with $99 \%$ specificity and $8 \%$ sensitivity for detection of Pat 1 subclinical IMI episodes. 
The predicted relationships between a preexisting subclinical IMI or EMR threshold and subsequent cases of the same condition in other cows are shown in Table 3 for each pathogen and EMR threshold. For several mastitis pathogens, the number of existing infections had a significant effect on the transmission risk from an infected cow to a susceptible uninfected cow. However, the only Pat 1 mastitis pathogens among these were Staph. aureus and Staph. epidermidis.

For the patterns of OCC change, the EMR threshold at 80,90 , and $99 \%$ specificity for Pat 1 subclinical IMI had a significant effect on whether this pattern would subsequently arise in another cow $(P=0.009,0.011$, and 0.009 , respectively).

\section{Numerical Simulations}

The proportion of cows with alerts for Pat 1 subclinical IMI based on the EMR are shown for the 4 levels of specificity by days of study, as obtained from the raw data in Figure 2. This curve shows the proportion of infected cows throughout the study period. The numerical simulations of the dynamics of I and $\mathrm{S}$ cows for each level of specificity are presented in Figure 2. Both the raw data and the dynamic simulations showed a stable transmission dynamic of the 4 patterns of OCC change in this herd throughout the study period. The numerical resolution of the ordinary differential equations describing the model can be used to generate predictions for any given time.

\section{DISCUSSION}

In this study, we propose using a transmission model based on frequent OCC measurements to forecast subclinical IMI dynamics at the herd level. By modeling patterns of OCC change, we are able to predict the evolution of OCC patterns, as a proxy for subclinical IMI, in the herd. We used EMR as described by $\mathrm{S} \varnothing-$ rensen et al. (2016) for this purpose. Changes in the proportions of $\mathrm{S}$ to I can be simulated and forecast for a prolonged period. This approach could be included in a decision-support tool to alert farmers when udder health management actions against subclinical IMI are required during lactation and at drying off.

Based on research, significant improvements have been made in detection, management, and prevention of mastitis (Ruegg, 2017). We propose further improvement in prevention of new cases of mastitis by using herd-specific evolution of the different transmission pa-

Table 2. Average duration in days of infection with subclinical IMI for individual and grouped (Pat 1 and Pat 2) mastitis pathogens and for 4 online cell count (OCC) patterns with different levels of specificity for detection of Pat 1 subclinical IMI episodes

\begin{tabular}{|c|c|c|}
\hline Subclinical IMI with pathogen & $\mathrm{N}^{1}$ & Duration $(95 \% \mathrm{CI})$ \\
\hline Staphylococcus epidermidis & 64 & $125(90-172)$ \\
\hline Corynebacterium bovis & 70 & $135(93-192)$ \\
\hline Staphylococcus chromogenes & 36 & $263(161-435)$ \\
\hline Staphylococcus aureus & 33 & $128(81-200)$ \\
\hline Staphylococcus haemolyticus & 22 & $161(95-270)$ \\
\hline Aerococcus viridans & 21 & $49(29-83)$ \\
\hline $\begin{array}{l}\text { Enterococcus faecalis, Enterococcus faecium, } \\
\text { and Lactococcus lactis }\end{array}$ & 12 & $455(172-1,250)$ \\
\hline Streptococcus dysgalactiae & 25 & $130(77-222)$ \\
\hline Staphylococcus simulans & 6 & $217(81-588)$ \\
\hline Staphylococcus hominis & 6 & $41(17-99)$ \\
\hline Streptococcus uberis & 7 & $345(86-1,429)$ \\
\hline Staphylococcus xylosus & 2 & $116(29-476)$ \\
\hline Other & 20 & $72(42-125)$ \\
\hline Pat 1 & 106 & $208(156-278)$ \\
\hline Pat 2 & 147 & $143(115-179)$ \\
\hline Elevated mastitis risk (EMR) $80 \%$ specificity $^{2}$ & 1,116 & $11(11-12)$ \\
\hline EMR $85 \%$ specificity $^{3}$ & 1,051 & $10(9-10)$ \\
\hline EMR $90 \%$ specificity $^{4}$ & 1,045 & $7(7-8)$ \\
\hline EMR $99 \%$ specificity $^{5}$ & 261 & $4(3-4)$ \\
\hline
\end{tabular}

${ }^{1}$ Number of subclinical IMI episodes.

${ }^{2}$ Pattern of OCC change with $80 \%$ specificity and $69 \%$ sensitivity for detection of Pat 1 subclinical IMI episodes.

${ }^{3}$ Pattern of OCC change with $85 \%$ specificity and $59 \%$ sensitivity for detection of Pat 1 subclinical IMI episodes.

${ }^{4}$ Pattern of OCC change with $90 \%$ specificity and $48 \%$ sensitivity for detection of Pat 1 subclinical IMI episodes.

${ }^{5}$ Pattern of OCC change with $99 \%$ specificity and $8 \%$ sensitivity for detection of Pat 1 subclinical IMI episodes. 
Table 3. Effect of an existing subclinical IMI episode or online cell count (OCC) pattern on the number of subsequent new events (transmissions of the same condition from infected cows to susceptible cows) ${ }^{1}$

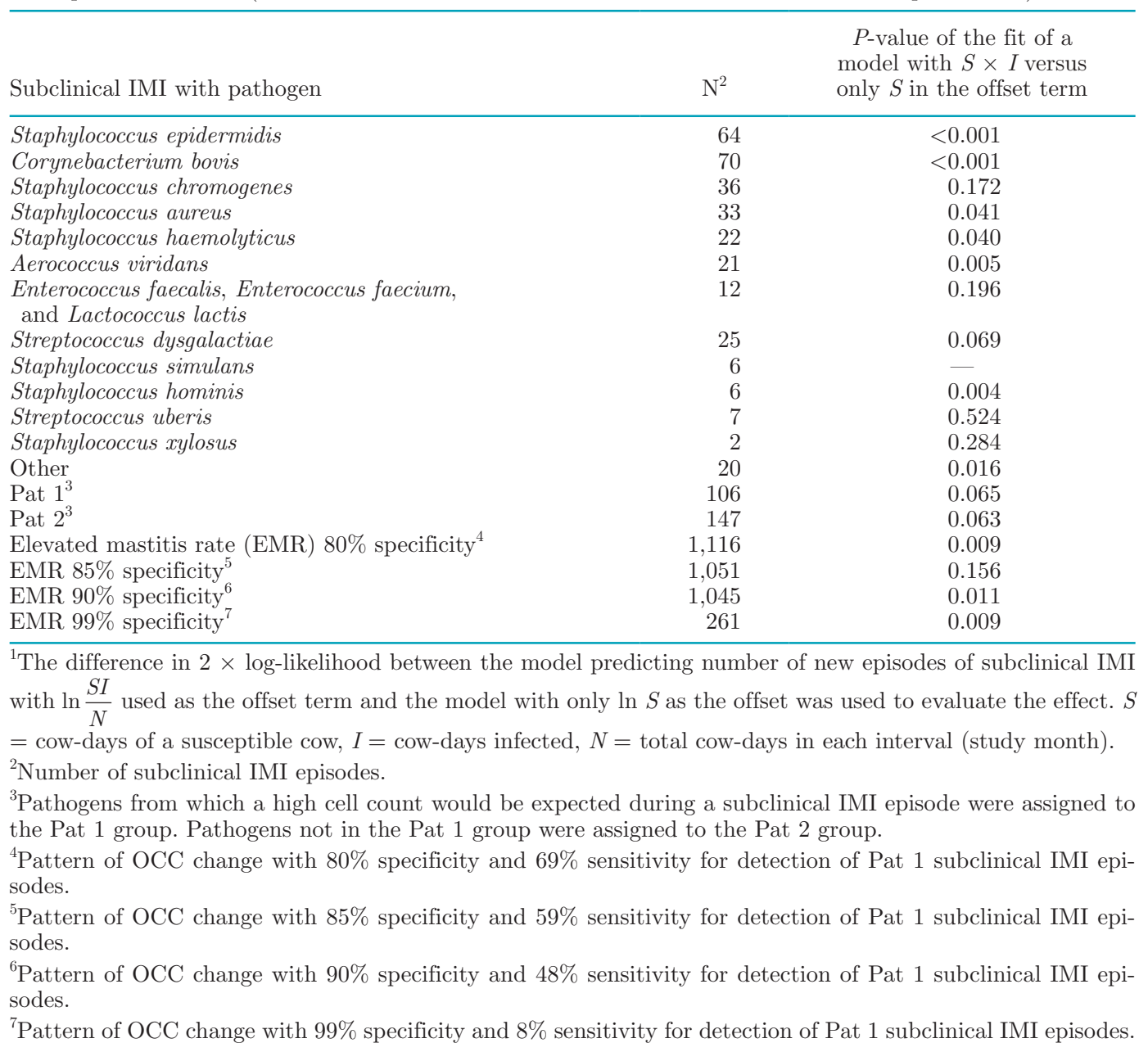

rameters to indicate which area of management should be improved to prevent an increase in new subclinical IMI episodes. Such transmission models also allow prediction of the effect of culling and treatment decisions on new cases of subclinical IMI, as demonstrated by Reksen et al. (2012).

An interesting finding in our study was the rather low transmission of Pat 1 mastitis pathogens between lactating cows. None of the Pat 1 pathogens demonstrated an $\mathrm{R}_{0}>1$. Also, but not unexpectedly, Staph. aureus and Staph. epidermidis were the only Pat 1 pathogens for which an existing subclinical IMI episode was significantly related to transmission from infected to susceptible cows. That is, existing infections with these pathogens were still transferred from one cow to another but at a lower rate than would be the case if $\mathrm{R}_{0}$ was $>1$. With only 2 AMS available for milking the cows in our study herd, we expected a higher degree of transmission of pathogens such as Staph. aureus and Strep. dysgalactiae between cows. In an AMS, some of the recommended preventive actions to limit transmission of contagious mastitis pathogens during milking (Barkema et al., 2009) are violated, because a large number of cows are milked with the same teat cups and the teat cups are only rinsed with lukewarm water between milkings. However, the observed absence of contagious properties of Staph. aureus and Strep. dysgalactiae in our study indicate that the AMS is not a major vector of transmission of subclinical IMI in this herd. In line with this, a previous study suggested reduced overmilking and no cross-quarter contamination in AMS as potentially beneficial factors for udder health in AMS (Hogeveen et al., 2001). This may explain the low transmission rates, although this is beyond the scope of the current study. Another potential explanation for this minor rate of transmission is that shedding of bacteria may be too low to enable effective transmission from cows with no clinical symptoms. Furthermore, the duration of subclinical IMI was short for most bacterial species, with the exception of Staph. 

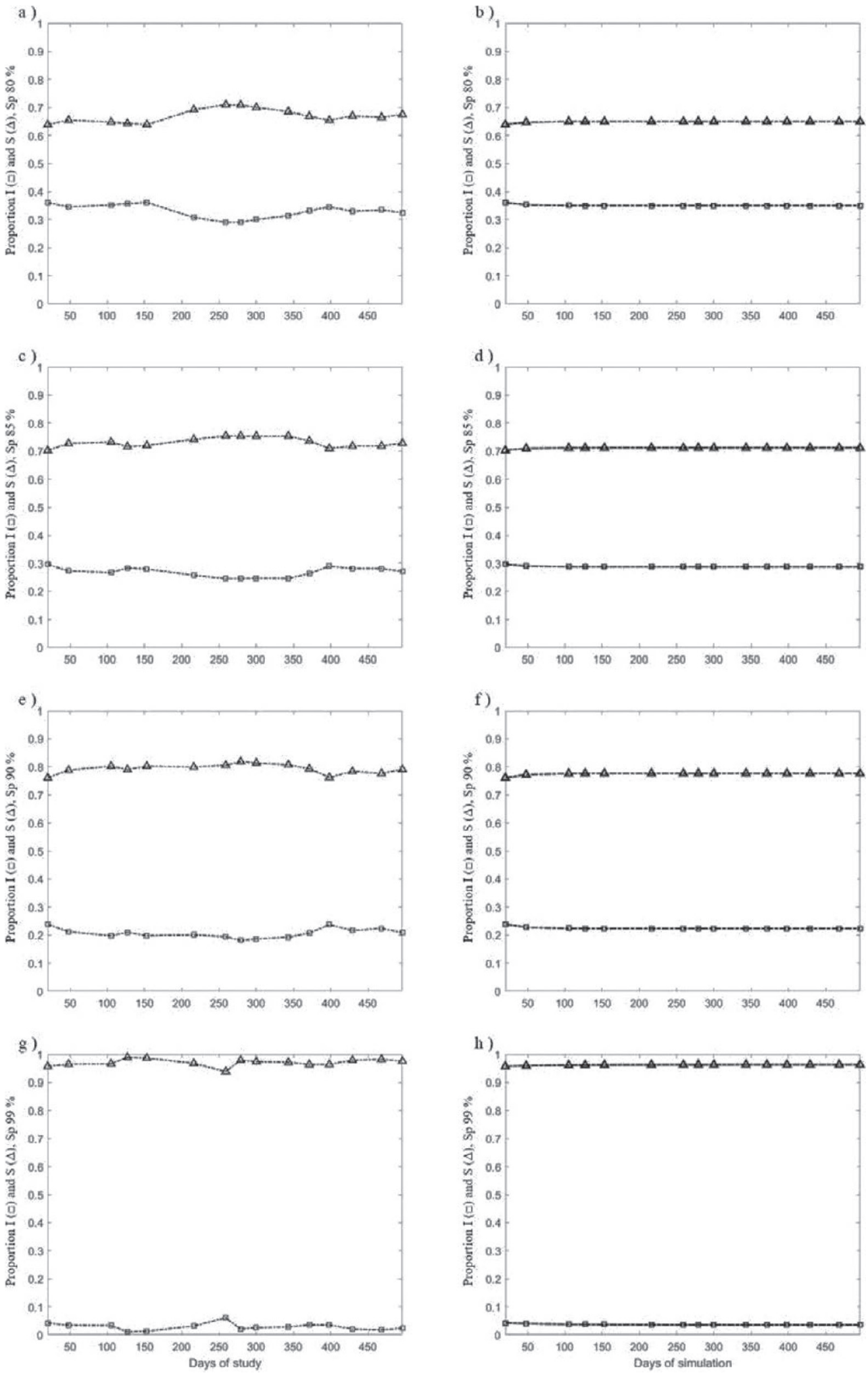

Figure 2. Observed proportion of cows with elevated mastitis risk (EMR) above the threshold (I; $\square$ ) and susceptible cows (S; $\Delta$ ) for specificity (Sp) of (a) 80\%, (c) 85\%, (e) 90\% and (g) $99 \%$ for Pat 1 (pathogens from which a high cell count would be expected during subclinical IMI) subclinical IMI using EMR. Corresponding dynamic simulation is shown for specificity of (b) $80 \%$, (d) $85 \%$, (f) $90 \%$, and (h) $99 \%$, respectively, for Pat 1 subclinical IMI. 
epidermidis and $C$. bovis. The high prevalence of $C$. bovis is likely to have elevated the transmission of this infection.

None of the known mastitis pathogens in our study demonstrated both an $\mathrm{R}_{0}>1$ during lactation and a significant effect of an existing subclinical IMI on transmission from infected to susceptible cows. This indicates that a major outbreak of mastitis cases due to these species of bacteria was unlikely. However, although transmission rates were low, an existing subclinical IMI was significantly associated with the number of new episodes of subclinical IMI for several Pat 2 pathogens, along with Staph. aureus and Staph. epidermidis. In such circumstances, the maintenance of a relatively constant prevalence of subclinical IMI is likely to depend on infected cows entering the lactation pen after calving (Reksen et al., 2012).

The EMR thresholds with 80 and $90 \%$ specificity for detection of Pat 1 subclinical IMI episodes showed both an $\mathrm{R}_{0}>1$ and a significant association between an existing pattern of OCC change and the occurrence of a new subclinical IMI episode in susceptible cows in our study. That is, the prevalence of cows with EMR over the threshold was significantly related to new episodes of EMR over the threshold in susceptible cows. In addition, spread of the patterns of OCC change apparently has the potential to be associated with an outbreak. This dynamic was observed despite no outbreak occurring during the study period; this implies, therefore, that this approach may be quite sensitive and useful for surveillance of the underlying udder health situation at the herd level. Our results also showed that the number of cows with an EMR over the threshold should be maintained at a low level to prevent an EMR over the threshold developing in other cows.

With low sensitivity and specificity for IMI, current application of sensors is of limited practical use for subclinical IMI management at the cow level (Norberg et al., 2004; Dalen et al., 2019). However, the current study showed that modeling transmission dynamics, based on patterns of OCC change as a proxy for subclinical IMI prevalence at herd level, may be useful for predicting the trend of new infections at the herd level. A forecast of an elevation in the proportion of infected cows in the herd may signal an increasing udder health problem in the herd. The usefulness of such a system depends on the defined threshold values for an alert. Application of predictions from the EMR-based transmission model with a specificity of $99 \%$ for Pat 1 subclinical IMI will result in relatively few alerts, but these will almost certainly be related to an ongoing subclinical IMI episode. This could prove useful for alerting the farmer of individual cows in need of attention. Lower- ing the specificity to $80 \%$ would result in more frequent but less specific alerts. These frequent alerts could be used in a surveillance of udder health on the herd level.

The duration of subclinical IMI episodes was significantly shorter for the 4 patterns of OCC change than the duration of the Pat 1 subclinical IMI episodes, as defined by culture results from QMS. A potential explanation for this is that the limited sensitivity and specificity for detection of Pat 1 subclinical IMI using the 4 patterns of OCC change results in a greater number of both false-positive and false-negative results. If so, this increases both the observed number of new episodes and cures, which, in turn, reduces the duration of each episode. Another possibility is that the duration of subclinical IMI episodes based on culture results is overestimated in our study, because milk sampling for bacteriological culture was performed monthly and the cows may have recovered from the infections in the period between the sampling events.

The basic reproduction number $R_{0}$ is a combined value affected by the number of contacts per unit time, transmission probability per contact, and duration of the infectious period (Anderson and May, 1991). Dalen et al. (2018) found that the cure rate for subclinical IMI differed significantly between 2 farms with the same mastitis pathogen, and that this difference affected the prediction of transmission dynamics of the same pathogen in each farm. With herd-specific knowledge of which transmission parameters have most effect on transmission dynamics, we can improve udder health management by focusing preventive actions on those management areas that are related to the transmission parameters of concern for each specific herd.

\section{CONCLUSIONS}

In the current study, we presented an investigation of transmission dynamics of mastitis pathogens, pathogen groups, and related alterations in EMR in a single herd. Forecasting changes in the herd prevalence of subclinical mastitis can be achieved using dynamic transmission models based on patterns of OCC change. The statistical analyses demonstrated transmission of patterns of OCC change as a proxy for subclinical IMI at specificity levels of 80,90, and 95\%, and new episodes of EMR over the threshold were influenced by patterns of OCC change exceeding the EMR threshold at specificity of 80,90 , and $99 \%$. Although limitations were apparent, this study provides proof of concept that an EMR transmission model can be used at different levels of specificity for Pat 1 subclinical IMI episodes. This could be used for surveillance during lactation, depending on an individual farmer's herd-health man- 
agement goals and tolerance for false positives. Future developments in sensor technologies and data analyses are likely to improve sensor-based transmission models.

\section{ACKNOWLEDGMENTS}

The authors acknowledge the contribution of participating farm staff. The support of our field and laboratory staff is also greatly appreciated. The data for this study were available from the project "New approaches to management and breeding, in automatic milking systems." The financial sources were TINE SA (Ås, Norway), DeLaval (Tumba, Sweden), Geno SA (Hamar, Norway), the Norwegian Research Funding for Agriculture and the Food Industry (Oslo, Norway), and the Norwegian Research Council (Lysaker, Norway).

\section{REFERENCES}

Anderson, R. M., and R. M. May. 1991. Infectious Diseases of Humans: Dynamics and Control. Oxford Science Publications, Oxford University Press, Oxford, UK.

Barkema, H. W., M. J. Green, A. J. Bradley, and R. N. Zadoks. 2009 Invited review: The role of contagious disease in udder health. J. Dairy Sci. 92:4717-4729.

Berry, D. P., and W. J. Meaney. 2006. Interdependence and distribution of subclinical mastitis and intramammary infection among udder quarters in dairy cattle. Prev. Vet. Med. 75:81-91.

Cheuzeville, L. 2015. Rapid detection of microorganisms in the dairy value chain by MALDI-TOF MS. MS Thesis. University of Stavanger, Stavanger, Norway.

Dalen, G., A. Rachah, H. Nørstebø, Y. H. Schukken, Y. T. Gröhn, J. W. Barlow, and O. Reksen. 2018. Transmission dynamics of intramammary infections caused by Corynebacterium species. J. Dairy Sci. 101:472-479.

Dalen, G., A. Rachah, H. Nørstebø, Y. H. Schukken, and O. Reksen 2019. The detection of intramammary infections using online somatic cell counts. J. Dairy Sci. 102:5419-5429.

Djabri, B., N. Bareille, F. Beaudeau, and H. Seegers. 2002. Quarter milk somatic cell count in infected dairy cows: A meta-analysis. Vet. Res. 33:335-357.

Dormand, J. R., and P. J. Prince. 1980. A family of embedded RungeKutta formulae. J. Comput. Appl. Math. 6:19-26.

Fry, P. R., J. R. Middleton, S. Dufour, J. Perry, D. Scholl, and I. Dohoo. 2014. Association of coagulase-negative staphylococcal species, mammary quarter milk somatic cell count, and persistence of intramammary infection in dairy cattle. J. Dairy Sci. 97:4876-4885.

Hogan, J. S., R. N. González, S. C. Harmon, S. C. Nickerson, J. W. Pankey, and K. L. Smith. 1999. Laboratory Handbook on Bovine Mastitis. 1st ed. National Mastitis Council, Madison, WI.

Hogeveen, H., W. Ouweltjes, C. J. A. M. de Koning, and K. Stelwagen 2001. Milking interval, milk production and milk flow-rate in an automatic milking system. Livest. Prod. Sci. 72:157-167.
Hyndman, R., A. Koehler, K. Ord, and R. Snyder. 2008. Forecasting with exponential smoothing: The state space approach. The State Space Approach. Springer, Berlin, Germany.

IDF. 2011. Suggested interpretation of mastitis terminology. Bull. IDF. Int. Dairy Fed., Brussels, Belgium.

Keeling, M. J., and P. Rohani. 2011. Modeling Infectious Diseases in Humans and Animals. Princeton University Press, Princeton, NJ.

Magal, P., and S. Ruan. 2008. Structured Population Models in Biology and Epidemiology. Vol. 1936. Lecture Notes in Mathematics. Springer, Berlin, Germany.

Norberg, E., H. Hogeveen, I. R. Korsgaard, N. C. Friggens, K. H. Sloth, and P. Løvendahl. 2004. Electrical conductivity of milk: Ability to predict mastitis status. J. Dairy Sci. 87:1099-1107.

Østerås, O., and L. Sølverød. 2009. Norwegian mastitis control programme. Ir. Vet. J. 62(Suppl. 4):S26-S33.

Otto, S. P., and T. Day. 2011. Biologist's Guide to Mathematical Modeling in Ecology and Evolution. Princeton University Press, Princeton, NJ.

Reksen, O., Y. T. Gröhn, J. W. Barlow, and Y. H. Schukken. 2012. Transmission dynamics of intramammary infections with coagulase-negative staphylococci. J. Dairy Sci. 95:4899-4910.

Reksen, O., L. Sølverød, and O. Østerås. 2008. Relationships between milk culture results and composite milk somatic cell counts in Norwegian dairy cattle. J. Dairy Sci. 91:3102-3113.

Ruegg, P. L. 2017. A 100-Year Review: Mastitis detection, management, and prevention. J. Dairy Sci. 100:10381-10397.

Sargeant, J. M., K. E. Leslie, J. E. Shirley, B. J. Pulkrabek, and G. H. Lim. 2001. Sensitivity and specificity of somatic cell count and California Mastitis Test for identifying intramammary infection in early lactation. J. Dairy Sci. 84:2018-2024.

Scherpenzeel, C. G., I. E. den Uijl, G. van Schaik, R. G. Riekerink, H Hogeveen, and T. J. Lam. 2016. Effect of different scenarios for selective dry-cow therapy on udder health, antimicrobial usage, and economics. J. Dairy Sci. 99:3753-3764.

Schukken, Y. H., D. J. Wilson, F. Welcome, L. Garrison-Tikofsky, and R. N. Gonzalez. 2003. Monitoring udder health and milk quality using somatic cell counts. Vet. Res. 34:579-596.

Simojoki, H., T. Orro, S. Taponen, and S. Pyörälä. 2009. Host response in bovine mastitis experimentally induced with Staphylococcus chromogenes. Vet. Microbiol. 134:95-99.

Simojoki, H., T. Salomäki, S. Taponen, A. Livanainen, and S. Pyörälä. 2011. Innate immune response in experimentally induced bovine intramammary infection with Staphylococcus simulans and S. epidermidis. Vet. Res. 42:49. Stata SE/14, Stata Corp., College Station, TX.

Sørensen, L. P., M. Bjerring, and P. Løvendahl. 2016. Monitoring individual cow udder health in automated milking systems using online somatic cell counts. J. Dairy Sci. 99:608-620.

White, L. J., T. J. Lam, Y. H. Schukken, L. E. Green, G. F. Medley, and M. J. Chappell. 2006. The transmission and control of mastitis in dairy cows: A theoretical approach. Prev. Vet. Med. 74:67-83.

Wood, P. D. P. 1967. Algebraic model of lactation curve in cattle Nature 216:164-165.

Zadoks, R. N., H. G. Allore, T. J. Hagenaars, H. W. Barkema, and Y. H. Schukken. 2002. A mathematical model of Staphylococcus aureus control in dairy herds. Epidemiol. Infect. 129:397-416. 